\title{
Thermodynamic model for a pilot balloon
}

2

\author{
Vicent Favà ${ }^{1}$, Juan José Curto ${ }^{1}$, Alba Gilabert ${ }^{2}$ \\ ${ }^{1}$ Observatori de l'Ebre, (OE) CSIC - Universitat Ramon Llull, Roquetes, Spain. \\ ${ }^{2}$ Centre for Climate Change, Department of Geography, Universitat Rovira i Virgili, Tarragona, Spain \\ Correspondence to: Juan José Curto (jjcurto@obsebre.es)
}

\begin{abstract}
In the early part of the 20th century, tracking a pilot balloon from the ground with an optical theodolite was one of the few methods that was able to provide information from the upper air. One of the most significant sources of error with this method, however, was involved in calculating the balloon height as a function of time, a calculation dependent on the ascent rate which was traditionally taken to be constant. This study presents a new thermodynamic model which allows us to compute the thermal jump between the surrounding environment and the lifting gas as a function of different parameters such as the atmospheric temperature lapse rate or the physical characteristics of the balloon. The size of the thermal jump and its effect on the ascent rate is discussed for a $30 \mathrm{~g}$ pilot balloon, which was the type used at the Ebro Observatory (EO) between 1952 and 1963. The meridional and zonal components of the wind profile from ground level up to $10 \mathrm{~km}$ altitude were computed by applying the model using EO digitized data for a sample of this period. The obtained results correlate very well with those obtained from the ERA5 reanalysis. A very small thermal jump with a weak effect on the computed ascent rate was found. This ascent rate is consistent with the values assigned in that period to the balloons filled with hydrogen used at the Ebro Observatory and to the $30 \mathrm{~g}$ balloons filled with helium used by the US National Weather Service.
\end{abstract}

Key words: Pilot balloon, Ascent rate, Thermodynamic model, Drag coefficient

\section{Introduction}

1.1 Pilot Balloon History

Pilot balloons were a widely used resource in the past to scan the wind regime at high altitudes in the atmosphere and may still sometimes be used today. With this method, winds aloft are measured by releasing a free, buoyant balloon, and following its motion by optical methods or other means. Synoptic-scale processes have been successfully studied and modelled by utilizing balloon-measured wind values.

After the first untethered manned hot air balloon flight was carried out in 1783 in Paris with a balloon created by the Montgolfier brothers (Crouch, 2008), a new world of possibilities for human-carrying flight technology opened up. For this reason, it became immediately apparent that there was a need to know the wind direction and speed at high altitudes. At first, small paper balloons -pilot balloons or pibals- were released before a manned ascent in order to determine the probable direction of the flight. Several years later, in 1809, T. Forster was the first person to observe the drift of these balloons with a telescope so as to study the multiple air currents. In 1874, Paul Schreiber constructed an instrument to specifically track large free balloons to determine the wind's movements. In 1903, rubber balloons replaced paper balloons. They had the advantage of maintaining practically constant rates of ascent. With a predictable ascent rate available, wind measurements using pilot balloons began to be made systematically. In 1905, "right-angled" telescopes were available. They facilitated angle readings by having the axis of the eyepiece horizontal regardless of the elevation of the objective of the telescope (Meteorological Office, 1961; Knowles, 1969). During World War II, there was a great need to obtain information about the wind from the upper layers of the atmosphere, so it was a time of intense research into new technologies and the growth of radiosonde networks. Pilot balloons were still in use through the 1960s, but the introduction of radio-sounds and radar, which are easier to handle and not limited by the presence of cloud and fog, eventually took over from pilot balloons as a technique to measure wind speed. 
49 In 1923, the daily launch of pilot balloons at the Ebro Observatory began (Puig, 1927), promoted by the Spanish meteorological service (INM). This task continued until 1975, providing a valuable series of historical wind data at high altitudes of great climatic interest, which is yet to be fully exploited.
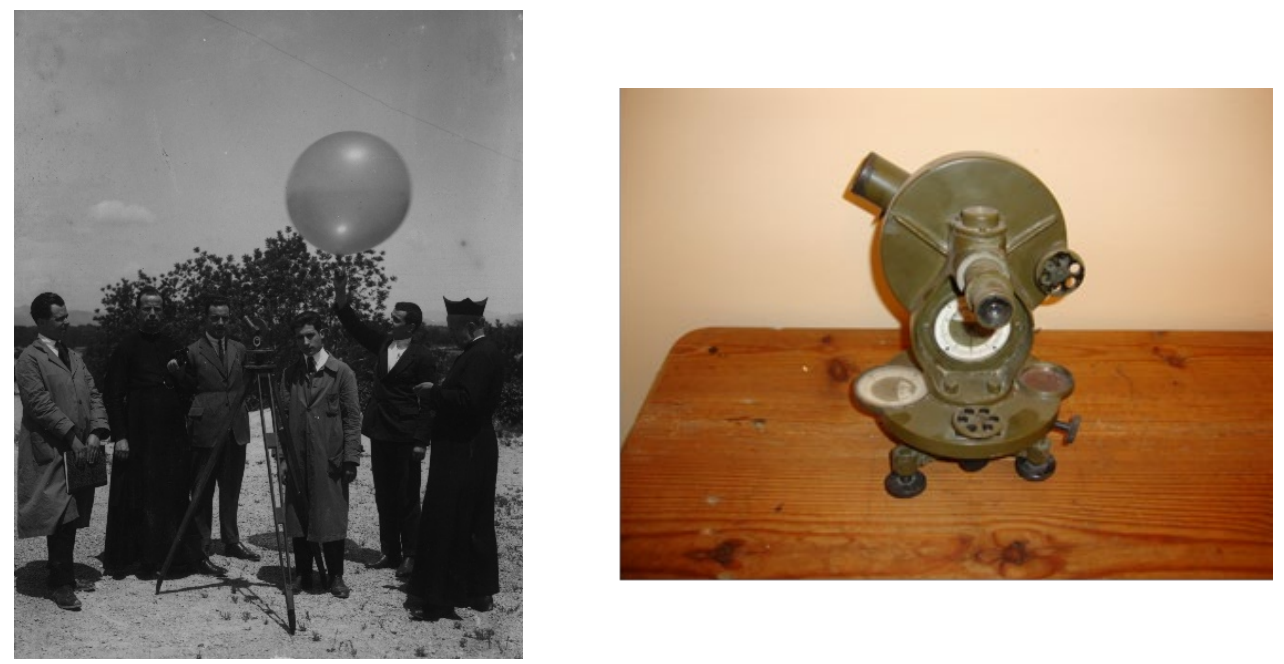

Figure 1. (Left) Fr. Rodes, director of the Ebro Observatory, and his assistants ready to lunch a pilot balloon. One assistant will track the balloon with a bent axis telescope theodolite mounted on a tripod while another assistant with a chronometer for a precise timing of the reading is ready to take notes of the angular positions of the balloon.

Figure 2. (Right) Theodolite for balloon tracking used in Ebro Observatory. Constructed by Hartmamm \& Braun (Frankfurt). Every morning (at 7 o'clock until 1957 and at 11 o'clock from 1958 onward), a 30 g rubber balloon filled with hydrogen was released with a free lift of $140 \mathrm{~g}$ (Fig. 1). It was tracked using a single special optical theodolite (Fig. 2) until it went out of sight. This usually happened at a height of around a few thousand metres, but in exceptional conditions of visibility and weak wind, sometimes it was possible to track it beyond $15 \mathrm{~km}$. Angular measurements of the position of the balloon in horizontal coordinates (elevation and azimuth) were recorded at regular intervals.

Balloons, at that time, were inflated with hydrogen, a very light gas that was easier to obtain than helium, although it is also more dangerous to use because it is very flammable.

The data obtained at the Ebro Observatory were sent daily to the Observatorio Meteorológico de Madrid and to Civil Navigation and were used by the pioneers of regular airlines such as the Latécoère airport, whose famous pilot on the Toulouse-Rabat route, Antoine de Saint-Exupéry, wrote brilliant pages in the history of air navigation (Saint-Exupéri, 1929).

\subsection{Background}

Over time, several studies have been conducted to determine the aerodynamic conditions of pilot balloons. For instance, 
the atmospheric layer through which the actual winds are averaged to obtain the balloon-measured wind. He discussed the influence of some of the balloon parameters on the effective thickness. Mapleson (1954) provided an empirical relation between the drag coefficient, $C d$, and the Reynolds number, $R e$, for captive, nominally spherical, rubber balloons. He suggested that the drag increases as the shape of the balloon progressively deviates from a spherical shape. Scoggins (1964 and 1965) showed that surface roughness of a spherical balloon stabilizes the drag force vector by reducing spurious aerodynamically horizontal motions, and the balloon measures the true wind more accurately. Fichtl et al. (1972) studied the fluctuations of the lift and drag coefficients associated with the aerodynamically induced motions of rising and falling spherical wind sensors. In agreement with Scoggins (1967), they found that the observed aerodynamically induced motions are predominantly horizontal, and concluded that the first-order rms (root mean square) drag coefficient is very small compared to the horizontal rms lift coefficient. Luers \& MacArthur (1974) and Jasperson (1982) estimated the wind accuracy that can be achieved by rising balloons. They evaluated errors due to tracking and probe balloon systems. Boatman (1974) discussed the effect of the tropospheric lapse rate on the ascent rate of pilot balloons for a variety of atmospheric temperature structures. He found that ascent rates through an isothermal layer would approach zero. However, Nelson (1975) found some discrepancies in these results and pointed out some explanations for this. He stated that in environments with a weak or negative temperature lapse rate, such as the case of isothermal layers or inversion layers, a faster increase in ascent rate with height would be expected, which is consistent with the conclusions of our study. Terliuc (1983) studied the problem of a loss of buoyancy due to gas leakage in balloons. Conrad (1991) showed that the existing balloon performance models produced inconsistent predictions of the ascent rate and the time needed to ascend to float altitude, and exhibited dependence on balloon size. Alexander (2003) pointed out that features during ascent depend on wind, small-scale air turbulence, and perturbations to the background atmosphere. He found approximate analytical solutions in certain cases and evaluated the effect of nonlinear drag on balloon oscillation periods and damping near flotation. Cross (2007) investigated the Magnus force on a spinning balloon. Gallilce et al. (2011) produced a model to describe the ascent of sounding balloons taking into account both the variation of the drag coefficient with altitude and the heat imbalance between the balloon and the atmosphere. The relationship between drag coefficient and Reynolds number was derived from a dataset of a series of flights and the transfer of heat from the surrounding air into the balloon was accounted for by solving the radial heat diffusion equation inside the balloon. measuring the upper wind using pilot balloons in single-theodolite ascents is the uncertainty in the balloon's rate of ascent. An error in the rate of ascent results in a proportional error in the height of the balloon and, hence, as modified by elevation angle, a proportional error in wind speed (Guide to Meteorological Instruments and Methods of Observation, 2014). In this study we want to take a step forward by generating a comprehensive thermodynamic model that can allow us to calculate an altitude profile from which we will be able to determine the actual balloon height corresponding to each measurement time interval. This model takes into account the heat imbalance between the balloon and the surrounding air, and the expansion work of the lifting gas as well as the thermal jump derived from the adiabatic expansion, the atmospheric temperature lapse rate, the gas leakage by diffusion, the free lift and the mass and type of lifting gas (which

113 can be helium or hydrogen). It also takes into account the variation of the balloon drag coefficient with height. However,

114 in order to keep the model manageable, five major assumptions were made: firstly, we considered the pressure difference

115 between the lifting gas and the surrounding air to be negligible. Secondly, we did not consider the impact radiation may

116 have on the gas temperature, meaning our model is only applicable in conditions with no or very little sunshine, for 
example, very early in the morning. Thirdly, we assumed that both air and the lifting gas behave like an ideal gas. We

118 also assumed a spherical shape for the balloon and, finally, we maximized the calculation of the thermal jump assuming

119 the case of a sphere under free convection.

The observations at the Ebro Observatory from the 1950s and 60s that we are looking at were used to construct the altitude profile of the horizontal components of the wind by assuming a constant ascent rate of $200 \mathrm{~m} \mathrm{~min}^{-1}$. However, that is quite an approximate number. A major goal of this paper is to be able to precisely evaluate to what extent this estimate of the ascent rate is adequate, an aim which is even more important considering that the same ascent rate was also assumed for other observatories within the national territory even if they were working with pilot balloons of different sizes inflated to different free lifts. On the other hand, the US National Weather Service, for pilot balloons with very similar characteristics to the Ebro Observatory ones (30 g of weight and $139 \mathrm{~g}$ of free lift) but inflated with helium, used significantly different ascent rate values $\left(600 \mathrm{ft} \mathrm{min}^{-1}\right)$ (Boatman, 1974). Analysing the effects on the ascent rate when changing the lifting gas from hydrogen to helium and contrasting the result with the values used by the US National Weather Service is another key goal of this study.

131

\subsection{Composition of the paper}

This work is structured in several sections. The data used and the methodology are presented in section 2 . The reasons why the calculation of the altitude of the pilot balloon as a function of time is of critical importance in order to be able to determine with the utmost precision the components of the vertical wind profile are presented in section 3 . In the next sections, some of the relevant physical properties of the balloons are discussed and a thermodynamic model is developed. With all this, and looking at both kinds of lifting gases, hydrogen and helium, the ascent rate has been determined considering the previously calculated factors: the thermal time constant of the system, gas leakage by diffusion, the thermal jump and the variation of the drag coefficient, $C d$, as a function of height. In the penultimate section, with a sample made up of 10 days when the balloons from EO reached an altitude higher than $10 \mathrm{~km}$, we compare the vertical wind profiles obtained from the ERA5 reanalysis with those computed assuming different constant ascent rates and with those assuming the variable ascent rate calculated with the thermodynamic model. The last section presents our conclusions.

\section{Data and methods}

The recent digitization of the data provided by pilot balloons at the Ebro Observatory (Fig. 3) was carried out using the 'manual keying' method (Wilkinson et al., 2019). Although it is a slower process, it provides much better results than the OCR (Optical Character Recognition) method for hand-written documents (as is the case of the pilot balloon data). We followed the 'key as you see' method, introducing the values directly as read from the original source dependent on the characteristics of the hand-writing and its state of conservation. Most of the data could be read quite easily. To ensure the quality and accuracy of our digitization we compared daily data summaries with calculated ones. Furthermore, after the data on each page were entered, we checked some values randomly as an additional quality control. 


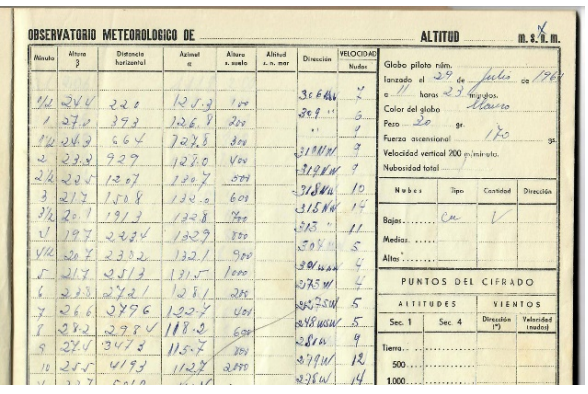

Figure 3: Copy of a fragment of the balloon records notebook corresponding to the initial measurements taken on 21 August 1961.

To calculate lineal regressions, as well as the least squares method, we used the Theil-Sen estimator. The Theil-Sen single median method (Theil, 1950; Sen, 1968) is very robust and less sensitive to the effects of outliers than the classic least squares method. The Theil-Sen estimator was calculated with the R software environment (R Core Team, 2020) using the package mblm (Komsta, 2013).

To contrast the results calculated with the instrumental observations, we used the ERA5 hourly data on pressure levels from 1950 to 1978 (preliminary version) (Bell et al., 2020) reanalysis. The ERA5 wind and geopotential data were obtained using Copernicus Climate Change Service information (2021). We chose this new reanalysis to obtain hourly data to provide high horizontal $(31 \mathrm{~km})$ and vertical (137 levels) resolutions.

The wind data from the two sources (observations and ERA5) refer to different altitudes so we carried out an interpolation process with the function of the NCL (The NCAR Command Language) csal with 15 knots (a large enough number to represent the original curves quite accurately).

\section{Tracking a pilot balloon using a theodolite}

175 Let $z_{i}$ be the pilot balloon's height and $\alpha_{i}, \beta_{i}$ its horizontal coordinates (elevation and azimuth, respectively) at time $t_{i}$. If $z_{i-1}, \alpha_{i-1}, \beta_{i-1}$ are the respective coordinates at $t_{i-1}$, and $\Delta t=t_{i}-t_{i-1}$, it can be shown by using basic trigonometry that the horizontal components of the balloon's velocity can be calculated with:

$$
\boldsymbol{u}_{i}=\frac{\frac{z i}{\tan \alpha_{i}} \cdot \cos \beta_{i}-\frac{z_{i-1}}{\tan \alpha_{i-1}} \cdot \cos \beta_{i-1}}{\Delta t} ; \quad \boldsymbol{v}_{i}=\frac{\frac{z_{i}}{\tan \alpha_{i}} \cdot \sin \beta_{i}-\frac{z_{i-1}}{\tan \alpha_{1-1}} \cdot \sin \beta_{1-1}}{\Delta t}
$$

These two formulas indicate that in order to calculate the Cartesian components of wind velocity with an optical theodolite, only using the observed angles (elevation and azimuth) does not give enough information. The exact height of the balloon must also be known. 
expected in the lower part of the atmosphere, a priori there is no physical reason to assume a constant ascent rate. Our

\section{Physical characteristics of the pilot balloons}

\subsection{Relative pressure inside the balloon}

The relative pressure inside a $30 \mathrm{~g}$ balloon, like the ones used at the EO, inflated with helium to give a free lift of $140 \mathrm{~g}$ (according to the specifications recorded by observers present at the launch of the balloons) was measured and seen to be that of an $8 \mathrm{~cm}$ column of water $(\approx 8 \mathrm{hPa}$ ). Even at an altitude of $10 \mathrm{~km}$, where the pressure is very low (around $300 \mathrm{hPa}$ ), the relative pressure of the balloon still only represents $2.6 \%$ of atmospheric pressure. Given this small relative pressure of the lifting gas, we assumed the internal pressure of the balloon is always equal to that of the surrounding air and we did not take it into account in our thermodynamic model when calculating the ascent rate.

\subsection{Gas loss by diffusion}

The thin rubber skin of the pilot balloon is highly permeable to the light filling gases commonly used (Terluic et al., 1983). This gas leakage contributes to reducing the buoyancy and the ascent rate during the ascent. Assuming that the diffusion of the gas is governed by Fick's Law, the buoyancy loss may be expressed as $Q=Q_{\infty} \exp \left(-\alpha T^{-1}\right)$ (Terliuc et al., 1983; Etherington, 1958). In this study we have used the parameters $Q_{\infty}$ and $\alpha\left(1.53 \times 10^{6} \mathrm{~g}^{\text {hour }}{ }^{-1}\right.$ and $3315 \mathrm{~K}$ respectively) found experimentally by Terluic et al. (1983), after re-dimensioning $Q_{\infty}$ since these authors worked with $300 \mathrm{~g}$ hydrogen balloons whereas we use $30 \mathrm{~g}$ ones. Assuming that the loss of lift by diffusion is proportional to the balloon's surface area, we divided the original $Q_{\infty}$ by a factor of 3.74, which is the relationship between the surface area of Terluic et al.'s $300 \mathrm{~g}$ balloons inflated to $1.1 \mathrm{~m}^{3}$ and the surface of the $30 \mathrm{~g}$ balloons used in our context.

Diffusion-caused gas losses decrease as balloons gain in height and the temperature drops. However, as pressure also drops at higher altitudes, the volume of the balloon expands together with its surface area. For this reason, we introduced a correction by multiplying parameter $Q_{\infty}$ at each altitude by a factor representing the proportion of the balloon's surface at that altitude with respect to its original surface area when launched. Unfortunately, though, we have not been able to correct for another possible factor - the fact that, as the balloon expands, its skin also becomes thinner which may increase to some extent the capacity of diffusion of the gas.

\subsection{Drag coefficient $\mathrm{Cd}$, how it varies as a function of altitude, and its impact on the evolution of the ascent rate} The aerodynamic force due to air friction that the balloon experiences as it rises is directly proportional to its transversal surface $(S)$, to the air density $(\rho)$, to the square of velocity $(v)$ and to the drag coefficient $(C d)$. It can be expressed as follows:

$$
F_{d}=0.5 C_{d} S \rho v^{2}
$$

As the balloon gains altitude, all of these parameters will vary to a greater or lesser degree. Pressure and density become lower, meaning the contact surface will increase as the balloon expands. Besides this, the drag coefficient will also vary as it depends on the Reynolds number, Re (equation 3), which, in turn, depends on the dynamic viscosity of air $(\mu$ ), the density $(\rho)$, the diameter of the balloon, and the ascent rate. The larger $R e$ is, the more turbulent the wind regime and the smaller the drag coefficient.

$$
R e=\frac{D \rho v}{\mu}
$$



in air density has a greater effect on it, meaning Re actually decreases with height. In fact, most kinds of pilot balloons can record critical and subcritical Reynolds numbers as they ascend, moving from a turbulent regime to a laminar one. This creates an abrupt change in the drag coefficient, making it impracticable to use a simple formula valid for balloons of different sizes and different free lifts (WMO-No.8, 2008). However, some types of balloons do not approach the critical zone at any stage of their flight, either because there is a laminar wind regime dominant throughout their ascent or because the regime is always turbulent. In these cases, we believe it is possible to find an empirical formula to relate the Reynolds number to the drag coefficient as Gallice et al. (2011) did.

The drag coefficient $(C d)$ is that of a homogeneous sphere whose movement is not impeded unlike the situation when wind tunnel experiments are used to measure the parameter. In our case, the balloon or sphere can experience significant horizontal movements due to the aerodynamic forces at play as it rises (Fichtl et al., 1972). These movements remove energy from the vertical motion which explains why the $C d$ for free ascent pilot balloons are higher than those calculated in wind tunnels for the same Reynolds number value (Scoggins et al., 1964).

In this study, to calculate the empirical relation between the drag coefficient and the Reynolds number, we used ascent rate data as a function of free lift for several types of balloons listed in the compendium of lecture notes for training class III (WMO, No 291, 1971, page 129, table 1.1). With the weight of the balloon, its free lift, and an estimate of its ascent rate, as given in the table, both the drag coefficient and Reynolds number can be calculated. For the $30 \mathrm{~g}$ pilot balloons used at the Ebro Observatory, the Reynolds number $(R e)$ calculated between 0 and $10 \mathrm{~km}$ of altitude varies approximately between values of $1.4 \times 10^{5}$ and $9 \times 10^{4}$. This interval of values, far from the critical zone, was used to find the $C d$ values in the previous table. The selected balloons have weights of $10 \mathrm{~g}, 30 \mathrm{~g}$, and $100 \mathrm{~g}$ with a free lift of $150 \mathrm{~g} .100 \mathrm{~g}$ balloons with larger free lift values and heavier balloons were not taken into account as they operate with much higher Re values. As there were few data corresponding to low $R e$ values in the previous table, we have also included $C d$ values calculated for Rose balloons by Scoggins (1965). A graph of drag coefficient in relation to the Reynolds number is shown in Fig. 4, according to the empirical formula we found:

$C_{d}=1.607871 \mathrm{E}-11 * R e^{2}-5.758793 \mathrm{E}-6 * R e+1.134552$

256

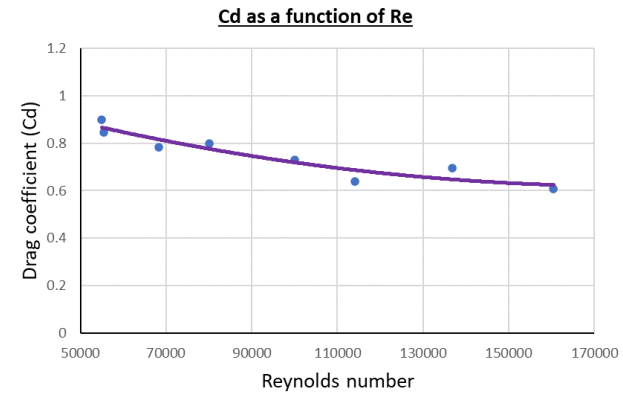




\section{Thermal model for a pilot balloon at rest}

In this section, we present a thermal model for a pilot balloon at rest which will serve as a starting point for the general model representing the behaviour of the balloon once it has been launched and is gaining altitude at a specific ascent rate. One of the main objectives of our study is to be able to estimate at all times the thermal jump between the surrounding air and the balloon's interior because any decrease in temperature of the lifting gas owing to adiabatic decompression and the resulting decrease in buoyancy could have a significant influence on the vertical ascent rate. That is why it is essential to determine what kind of dynamic behaviour the thermal response of the balloon shows when the balloon is forced to change its temperature with respect to the surrounding air. In this section we will study this dynamic behaviour for the specific case of a balloon at rest.

270

To study the thermodynamics of the balloon we analysed the behaviour of the lifting gas temperature of a balloon at rest when there is a sudden temperature change in the surrounding air. To this end, we inflated a balloon with helium to produce a free lift of $140 \mathrm{~g}$ (according to the specifications the EO observers followed at the launches carried out in the 1950s and 60s). Meteorological observers normally used to work with hydrogen at the Ebro Observatory but for safety reasons our experiment was carried out with helium. Next, the balloon was ballasted with a pay load of $240 \mathrm{~g}$. The pay load attached to the balloon weighed $100 \mathrm{~g}$ on precision scales at a room temperature of $22{ }^{\circ} \mathrm{C}$. The balloon was then placed in a chamber at $40^{\circ} \mathrm{C}$ and left there a few minutes until a thermal and radiative balance was achieved between the balloon and the heated chamber. Next, the balloon was taken out again and weighed at 10-second intervals. The aim of the experiment was to indirectly measure the internal gas temperature of the balloon at 10 -second intervals based on these weight measurements.

The free lift generated by the balloon (expressed in grams) can be obtained from the weight measurements:

$$
\text { measured_weight }=240-\text { Free_lift; }
$$

We also know that the free lift expressed in grams is the mass of displaced air $\left(\mathrm{m}_{\mathrm{a}}\right)$ less the mass of the helium ( $\left.\mathrm{m}_{\mathrm{he}}\right)$ less the mass of the balloon's rubber skin or envelope $\left(m_{g}\right)$ :

$$
\text { Free_lift }=m_{a}-\left(m_{h e}+m_{g}\right)
$$

The mass of the helium gas was calculated previously ( $27 \mathrm{~g}$ ) and is considered constant. The mass of the rubber balloon itself is $30 \mathrm{~g}$. Thus, following the two formulas mentioned above, we can calculate the mass of displaced air knowing the weight recorded in grams on the scales:

$$
m_{a}=240-\text { measured_weight }+m_{h e}+m_{g} ; \quad m_{a}=297-\text { measured_weight }
$$

The air density $\left(\rho_{a}\right)$ at the time of the experiment was $1.2 \mathrm{~kg} \mathrm{~m}^{-3}$ at a temperature of $22{ }^{\circ} \mathrm{C}$ and an atmospheric pressure of $1015 \mathrm{hPa}$. This allowed us to determine the volume $(V)$ of the mass of displaced air $\left(m_{a}\right)$ for each time interval. As this volume (ignoring the small volume made up of the balloon's rubber skin) is equivalent to that occupied by the helium, using the equation of state we were able to calculate the gas temperature every 10 seconds (the constant for 
Values obtained for the thermal jump at 10 -second intervals, calculated by subtracting room temperature $\left(22^{\circ} \mathrm{C}\right)$ from the calculated temperature $T_{h e}$ are shown in the graph in Fig. 5 (blue curves).

Heat transfer from the balloon's interior to the exterior (and vice versa) is influenced by different processes. Heat is propagated from the gas to the balloon's skin or envelope and then to the surrounding air by convection, conduction and radiation. Convection plays an important role within the gas and the only limiting factor is the skin of the rubber balloon. For this reason, we established a hypothesis that the thermal gradient inside the balloon is insignificant with respect to that between the temperature at the skin and the surrounding environment. We assumed a uniform temperature for the gas inside the balloon equal to the temperature at the skin and we also assumed that the thermal jump appears between

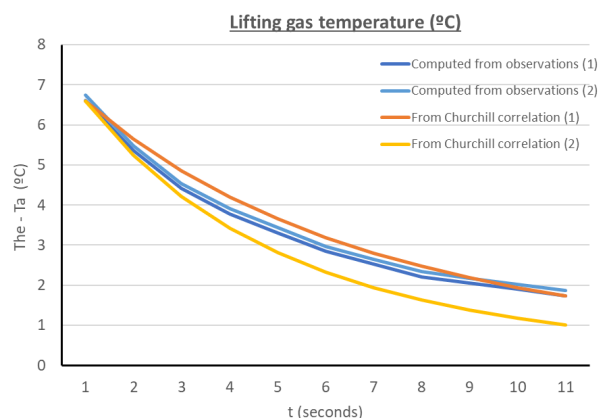

Figure 5:. Temperature difference between the lifting gas temperature, $T_{h e}$, and the environment, $T_{a}$, as a function of time. Computed from observations taking into account the weight system resolution (minimum possible values in dark blue (1) and maximum ones in light blue (2)). Temperatures computed from the Churchill correlation in red (1) and, without taking into account the thermal inertia of the balloon envelope, in orange (2).

In this way, the thermal resistance of the gas itself is taken to be relatively low and insignificant. The only thermal resistance we took into account is that representing the difficulty the exterior of the balloon's skin has in transferring heat to the outside. The thermal capacity of the system is the sum of the thermal capacity of the gas plus that of the rubber skin, as they can be considered to be two thermal condensers in parallel once we have assumed the thermal resistance of the gas has no importance (Fig. 6). 


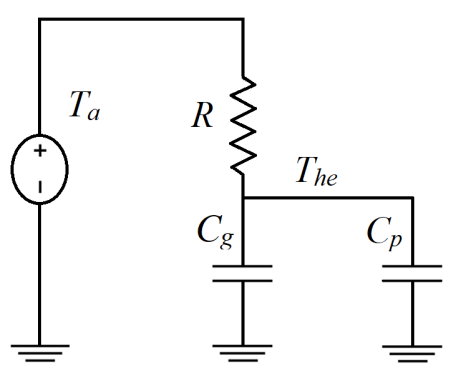

Figure 6: Thermal model for the balloon at rest

The total thermal capacity can be calculated by adding the thermal capacities $C_{g}$ (balloon skin capacity) and $C_{p}$ (gas capacity) obtained from the balloon's mass of $30 \mathrm{~g}$ and the $27 \mathrm{~g}$ of helium, and assuming specific heats of $2000 \mathrm{~J} \mathrm{~kg}^{-1}$ $\mathrm{K}^{-1}$ and $5200 \mathrm{~J} \mathrm{~kg}^{-1} \mathrm{~K}^{-1}$ for the balloon and helium, respectively, giving a value for the capacity $C$ of $200.4 \mathrm{~J} \mathrm{~K}^{-1}$. To calculate the thermal resistance, $R$, we applied the Churchill correlation (Churchill, 1983) for an isothermal sphere in conditions of free convection. The procedure used to calculate it is explained below.

The thermal jump between the balloon skin and the surrounding air divided by the thermal resistance between the balloon skin and air gives the thermal power (equation 9). This thermal power is proportional to the balloon's area (Sup) and the heat transfer coefficient $(h)$, according to Newton's law of cooling, which means we can calculate R:

341

342

$\frac{T_{h e}-T_{a}}{R}=h \operatorname{Sup}\left(T_{h e}-T_{a}\right)$

343

344

$R=\frac{1}{h \sup }$

Hence, thermal resistance is inversely proportional to the balloon's surface area and the heat transfer coefficient, h, but neither of these are constant values. The surface area increases due to decompression as the balloon ascends and the heat transfer coefficient depends on many factors, including the thermal jump itself. Having said that, we can determine $h$ with the Nussel number $(\mathrm{Nu})$, the lifting gas conduction coefficient $(k)$ and the diameter of the balloon $(D)$ :

For an isothermal sphere, according to the Churchill correlation and assuming external free convection, the Nusselt number is:

$$
N u=2+\frac{0.589 R a^{\frac{1}{4}}}{\left[1+\left(\frac{0.43}{P r}\right)^{\frac{9}{16}}\right]^{\frac{4}{9}}} \quad \text { if }\left(R a \leq 10^{11} ; P r \geq 0.7\right)
$$




$$
G r=\frac{D^{3} \rho^{2} g \Delta T \beta}{\mu^{2}} ; \operatorname{Pr}=\frac{\mu c_{p}}{k} ; R a=G r P r
$$

359
Where $D$ is the balloon diameter, $\rho$, the air density, $g$, the acceleration of gravity, $\Delta T$, the thermal jump, $\beta$, the fluid thermal expansion coefficient (the inverse of absolute temperature of the air surrounding the balloon), $\mu$, the dynamic viscosity of air, $C_{p}$, the thermal capacity of air at a constant pressure, and $G r$ is the Grashof number. These parameters allow us to calculate the heat transfer coefficient, $h$, with respect to altitude and, through equation 10 , the thermal resistance between the balloon skin and its surroundings at each atmospheric level.

When the balloon is launched from its rest state, it will rise at an ascent rate of around $3 \mathrm{~m} \mathrm{~s}^{-1}$, meaning convection will be forced rather than natural and so at any altitude the thermal resistance between the balloon's rubber skin and the surrounding air will be lower than that obtained via the Churchill correlation. Thus, the thermal jump will also be lower. However, as the ascent rate is relatively low, and given the lack of wind in high altitude observations (we can only follow the balloon's route at high altitude in meteorological situations of little or no wind), to calculate the thermal jump with respect to altitude, we maintained the hypothesis of an isothermal sphere and natural convection. This maximizes the calculation of the thermal jump between the gas and surrounding atmosphere and its potential effects on the balloon's ascent rate.

\section{Thermodynamics of a pilot balloon in free ascent}

\subsection{Equation of the balloon}

As the balloon gains height, the lifting gas will expand and absorb thermal energy from its surroundings, a part of which will be used for the expansion work. We shall now form an equation to calculate the expansion power of the balloon from its ascent rate.

With the equation of state, for the mass unit of the lifting gas, we have:

$$
p V=r_{\text {gas }} T_{\text {gas }} ; d(p V)=p d V+V d p=r_{\text {gas }} d T_{\text {gas }} ; \quad p \frac{d V}{d z}+V \frac{d p}{d z}=r_{\text {gas }} \frac{d T_{\text {gas }}}{d z}
$$

But $V$ is the specific volume of the gas inside the balloon. That is, the inverse of the gas density. Applying the hydrostatic equation, we have:

$$
V d p=\frac{1}{\rho_{g a s}}\left(-g \rho_{a} d z\right)=-\frac{\rho_{a}}{\rho_{g a s}} g d z
$$

Applying the equation of state again:

$$
V d p=-\frac{\rho_{a}}{\rho_{\text {gas }}} g d z=-\frac{\frac{p}{r_{a} T_{a}}}{\frac{p}{r_{\text {gas }} T_{\text {gas }}}} g d z=-\frac{r_{\text {gas }} T_{\text {gas }}}{r_{a} T_{a}} g d z
$$


However, as the difference between $T_{g a s}$ and $T_{a}$ is small, we can approximate to $\frac{T_{g a s}}{T_{a}} \approx 1$, and substitute it in equation 14:

$$
p \frac{d V}{d z}-g \frac{r_{\text {gas }}}{r_{a}} \frac{d z}{d z} \approx r_{g a s} \frac{d T_{\text {gas }}}{d z} ; p \frac{d V}{d z} \frac{d t}{d t}-g \frac{r_{\text {gas }}}{r_{a}} \approx r_{g a s} \frac{d T_{\text {gas }}}{d z} ; p \frac{d V}{d t} \frac{d t}{d z}-g \frac{r_{\text {gas }}}{r_{a}} \approx r_{g a s} \frac{d T_{\text {gas }}}{d z}
$$

This equation allows us to calculate the expansion work of the gas for each unit of time from the ascent rate $(v)$, the rate the gas temperature falls with altitude $\left(d T_{\text {gas }} / d z\right)$ and the gas constants (air and hydrogen).

403

404

\subsection{Generalized thermodynamic model for the pilot balloon}

Using the balloon equation explained above, we were able to develop a thermodynamic model to calculate the thermal jump as the balloon gains in altitude.

The first law of thermodynamics states that heat transferred to a gas is transformed into work and an increase in internal energy:

$$
d Q=C_{v} d T+p d V ; \quad \frac{d Q}{d t}=C_{v} \frac{d T}{d t}+p \frac{d V}{d t}
$$

We calculated the expansion work per unit of time in the previous section. It can be expressed with respect to the rate at which the gas temperature increases. If we start out from equation 17:

But if $R$ is the thermal resistance from the exterior of the balloon skin to the surrounding air, the heat per time unit reaching the balloon's interior must fulfil:

If we combine equations 18,19 , and 20 , and taking into account the capacity of the balloon skin, we see that:

423

$$
p \frac{d V}{d t} \approx g v \frac{r_{g a s}}{r_{a}}+r_{g a s} \frac{d T_{\text {gas }}}{d z} \frac{d z}{d t} ; \quad p \frac{d V}{d t} \approx g v \frac{r_{\text {gas }}}{r_{a}}+r_{g a s} \frac{d T_{\text {gas }}}{d t} ;
$$

$$
\frac{T_{a}-T_{g a s}}{R} \approx C_{v} \frac{d T_{\text {gas }}}{d t}+g v \frac{r_{\text {gas }}}{r_{a}}+r_{g a s} \frac{d T_{\text {gas }}}{d t}+C_{g} \frac{d T_{\text {gas }}}{d t}
$$




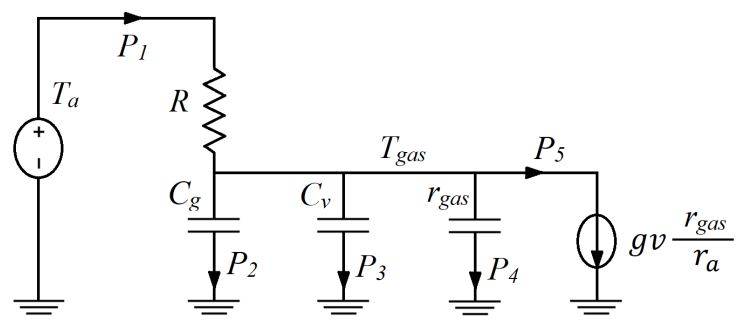

In this circuit, the temperature generator $T_{a}$ represents the temperature of the air surrounding the balloon, $R$ is the difficulty the heat flux comes up against when transferring from the surrounding environment to the interior gas, $C_{v}$ represents the specific heat of the gas at a constant volume, and the thermal capacitor $\left(r_{\text {gas }}\right)$ represents the difference between the enthalpy and the internal energy per mass unit. The generator of thermal power proportional to the ascent rate of the balloon represents the energy per time unit and mass unit the interior gas will lose due to decompression $(=-V d p / d t$, where $V$ is the specific volume of the gas). We can consider the ascent rate to be constant over a very short period of time so, although the rate at which pressure falls becomes lower and lower (being proportional to air density, $d p=-\rho g d z$ ), the rate at which the gas loses energy through decompression is constant because the volume of gas increases by the same proportion (inversely proportional to its density). For the case where hydrogen is used as the lifting gas, the expression $r_{\text {gas }} / r_{a}$ multiplies power by 14.37 with respect to a balloon full of air as $1 \mathrm{~kg}$ of hydrogen occupies a volume 14.37 times larger than that of $1 \mathrm{~kg}$ of air at the same pressure and temperature.

The sum of the powers of the two branches on the right $\left(P_{4}\right.$ and $\left.P_{5}\right)$ is equal to the thermal power which will be converted into the expansion power developed by the gas inside the balloon as it gains altitude. The origin of this power will depend on the kind of thermodynamic process the gas undergoes. As we shall see, this process depends on the lapse rate.

In this model, both the exterior temperature, $T_{a}$, and the gas temperature, $T_{g a s}$, as well as the ascent rate, will depend on altitude. The other parameters are taken as constant.

\subsection{Analytical solution of the differential equation. Calculation of the thermal jump in steady state}

One of the principal objectives of the model was to calculate the thermal jump (defined from now on as the temperature difference between the surrounding air and the lifting gas) as a function of altitude because this may affect the vertical ascent rate of the balloon. Looking at it from a mathematical analysis point of view allows us to derive equations which are very useful for estimating the size of the thermal jump for different scenarios of vertical temperature gradient (for example, the case of a vertical profile equal to the dry air adiabatic lapse rate, or for the case of an isothermal atmosphere). resistance. This gives:

$$
-\frac{\partial T}{\partial z}=\text { constant }=\alpha ; T_{a}=T_{a 0}-\propto z ; \quad \text { but } \quad z=z_{0}+v t
$$


If $\tau=R\left(C_{p}+C_{g}\right)$, the solution for this differential equation, for mass unit, after introducing $z_{0}=0$ and changing the variable $t=z / v$ will be:

$$
T_{g a s}=-\propto\left[z-v \tau\left(1-e^{\frac{-z}{v \tau}}\right)\right]-R g v \frac{r_{g a s}}{r_{a}}\left(1-e^{\frac{-z}{v \tau}}\right)+T_{g a s 0}
$$

During the first moments after launch, the process is adiabatic as there will have been no time to establish a temperature difference between the balloon's interior and exterior, meaning there will be no heat flux. Later, when the process reaches a stationary regime, the temperature in the balloon will vary with altitude at the same rate as the vertical gradient if this is constant.

From equation 24, and assuming $T_{g a s}=T_{a 0}$, it can be determined that the thermal jump between the surrounding air and the lifting gas for mass $m_{\text {gas }}$ of gas in steady state, will be:

$$
T_{a}-T_{g a s}(t \rightarrow \infty)=m_{g a s} v R\left(g \frac{r_{g a s}}{r_{a}}-\propto C_{p}-\propto C_{g} \frac{m_{g}}{m_{\text {gas }}}\right)
$$

This equation shows that the thermal jump will be larger, the greater the ascent rate, the thermal resistance, and the mass of the gas inside the balloon. It also shows that the smaller the lapse rate is, for example in an isothermal atmosphere or inversion layer, the larger the thermal jump. On the other hand, for large lapse rates, weaker thermal jumps will be found; for example, for a dry air adiabatic lapse rate $\left(g / C_{p a}\right)$, there will be no thermal jump if the lifting gas is hydrogen and the capacity of the balloon skin is taken to be insignificant.

For the case of a dry adiabatic lapse rate and neglecting the skin's capacity, we have:

$$
T_{a}-T_{g a s}(t \rightarrow \infty)=m_{g a s} v R\left(g \frac{r_{g a s}}{r_{a}}-g \frac{C_{p g a s}}{C_{p a}}\right)
$$

For the case of a diatomic lifting gas, such as hydrogen, the former subtraction gives a result of zero,

because, as air is principally diatomic too, then $C p=7 r / 2$ holds true for both gases. However, if the lifting gas is monatomic, like helium, then the $\mathrm{Cp}$ for helium would be $C p=5 r / 2$ and, for this case, the steady state thermal jump would be:

$$
T_{a}-T_{\text {gas }}(t \rightarrow \infty)=m_{\text {gas }} v R g \frac{2}{7} \frac{r_{\text {gas }}}{r_{a}}
$$

If the lifting gas is helium, for the same lapse rate conditions, the thermal jump is larger than that of the case with hydrogen as, being a monatomic gas, its capacity for storing internal energy is lower and its adiabatic lapse rate is greater ( $7 / 5$ with regards to dry air). Thus, for there to be no thermal jump in the case of a helium balloon, the temperature lapse rate must be around $13.7 \mathrm{~K} \mathrm{~km}^{-1}$. 
The circuit in Fig. 7 helps to understand intuitively the reason why we expect to see a larger thermal jump in the case of small lapse rate values. Take the example of a case with isothermal layer. When steady state is reached, the lifting gas temperature will have settled at a constant value and, therefore, the powers $P_{2}, P_{3}$ and $P_{4}$ will be null which means all the generator's power $\left(P_{5}\right)$, will have to pass through the resistance, creating a high thermal jump. With a dry adiabatic lapse rate, the opposite occurs. In this case, if the gas is hydrogen, all the expansion power comes from the internal energy of the gas and, if we ignored the balloon skin's capacity, then $P_{4}+P_{5}=-P_{3}$, meaning both the resistance power $\left(P_{1}\right)$ and the thermal jump would be null. the lapse rate, nor the ascent rate, nor the thermal resistance between the skin and surrounding air are constant. For this reason, we have solved equation 21 using a numerical method.

\subsection{Numerical solution of the differential equation}

Taking into account the circuit in Fig. 7, if $m_{\text {gas }}$ and $m_{g}$ are the mass of the gas and the balloon's skin, respectively, and if $\bar{T}_{a}$ and $\bar{T}_{g a s}$ are the average values of $T_{a}$ and $T_{g a s}$ during time interval $\Delta t$, we can calculate the average power arriving from the surrounding air during this $\Delta t$ with the following equation:

With $T_{\text {gas } 2}$ in equation 28, we have:

$$
\frac{\bar{T}_{a}-\bar{T}_{g a s}}{R}=\left(m_{g a s} C_{p}+m_{g} C_{g}\right) \frac{\Delta T_{g a s}}{\Delta t}+m_{g a s} g v \frac{r_{g a s}}{r_{a}}
$$

If the time interval $\Delta t$ is short enough (in our case, 1 second), variations can be considered to be linear and we can apply the Trapezoidal Rule (Atkinson and Kendall, 1989):

$$
\bar{T}_{a}=\frac{T_{a 1}+T_{a 2}}{2} ; \quad \bar{T}_{g a s}=\frac{T_{g a s}+T_{g a s 2}}{2} ; \Delta T_{g a s}=T_{g a s 2}-T_{g a s 1}
$$

\section{Calculation of the balloon's vertical ascent rate}

If $m_{\text {gas }}$ is the mass of the gas, $m_{g}$ is the mass of the balloon (its skin) itself, and $v$ is the ascent rate of the balloon, at the launch moment the free lift (equation 6) will develop an acceleration which will slow as the drag force ( $F_{d}$, proportional 
A moment later, when the initial acceleration of the balloon has developed the ascent rate or velocity of a stationary regime, the free lift will end up in equilibrium with the drag force. Finally, if $m_{a}$ is the mass of displaced air, we see that:

$$
P_{a} V_{a}=P_{\text {gas }} V_{\text {gas }} ; \quad m_{a} r_{a} T_{a}=m_{\text {gas }} r_{\text {gas }} T_{\text {gas }} ; m_{a}=m_{\text {gas }} \frac{r_{\text {gas }}}{r_{a}} \frac{T_{\text {gas }}}{T_{a}} ;
$$

The lower the temperature of the gas with respect to the surrounding air, the less volume is displaced, meaning less displaced air mass and less lift. Finally, we can obtain the ascent rate as a function of the mass of the gas ( $\left.m_{\text {gas }}\right)$ and the interior $\left(T_{g a s}\right)$ and exterior temperatures $\left(T_{a}\right)$ of the balloon by combining equations 32 and 33 :

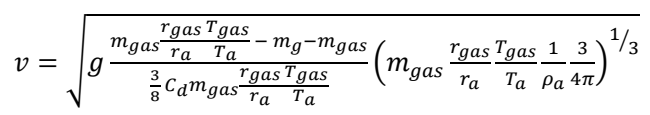

\section{Computing the air density}

To calculate the balloon's ascent rate using equation 34, first we need to determine the air density with regards to altitude.

$$
p=r \rho T ; \quad d p=r \rho d T+r T d \rho ; \frac{d p}{r \rho T}=\frac{d T}{T}+\frac{d \rho}{\rho}
$$

From the hydrostatic equilibrium we know that $d p=-g \rho d z$. Thus, the previous equation becomes:

$$
\frac{-g d z}{r T}=\frac{d T}{T}+\frac{d \rho}{\rho}
$$

If the lapse rate of temperature were constant, after integrating the previous equation, we would obtain the following formula for density: 
https://doi.org/10.5194/amt-2021-206

Preprint. Discussion started: 18 August 2021

(C) Author(s) 2021. CC BY 4.0 License.

575 Thus, if an increase in altitude $\Delta z\left(z_{2}-z_{l}\right)$ sees an increase in density $\Delta \rho\left(\rho_{2}-\rho_{l}\right)$, we will obtain the following

576 according to the Trapezoidal Rule:

577

$$
\Delta \rho=\left(\alpha-\frac{g}{r}\right) \frac{\Delta z}{T}\left(\frac{\rho_{1}+\rho_{2}}{2}\right)
$$

579

580 This equation indicates that, unless the lapse rate is larger than $g / r$ (seen very rarely, one example being close to a surface strongly heated by solar radiation), each positive increase in altitude corresponds to a negative increase in density.

582

\section{Outline of the thermodynamic model}

584 The thermodynamic model was implemented in a computer program structured in several steps. 


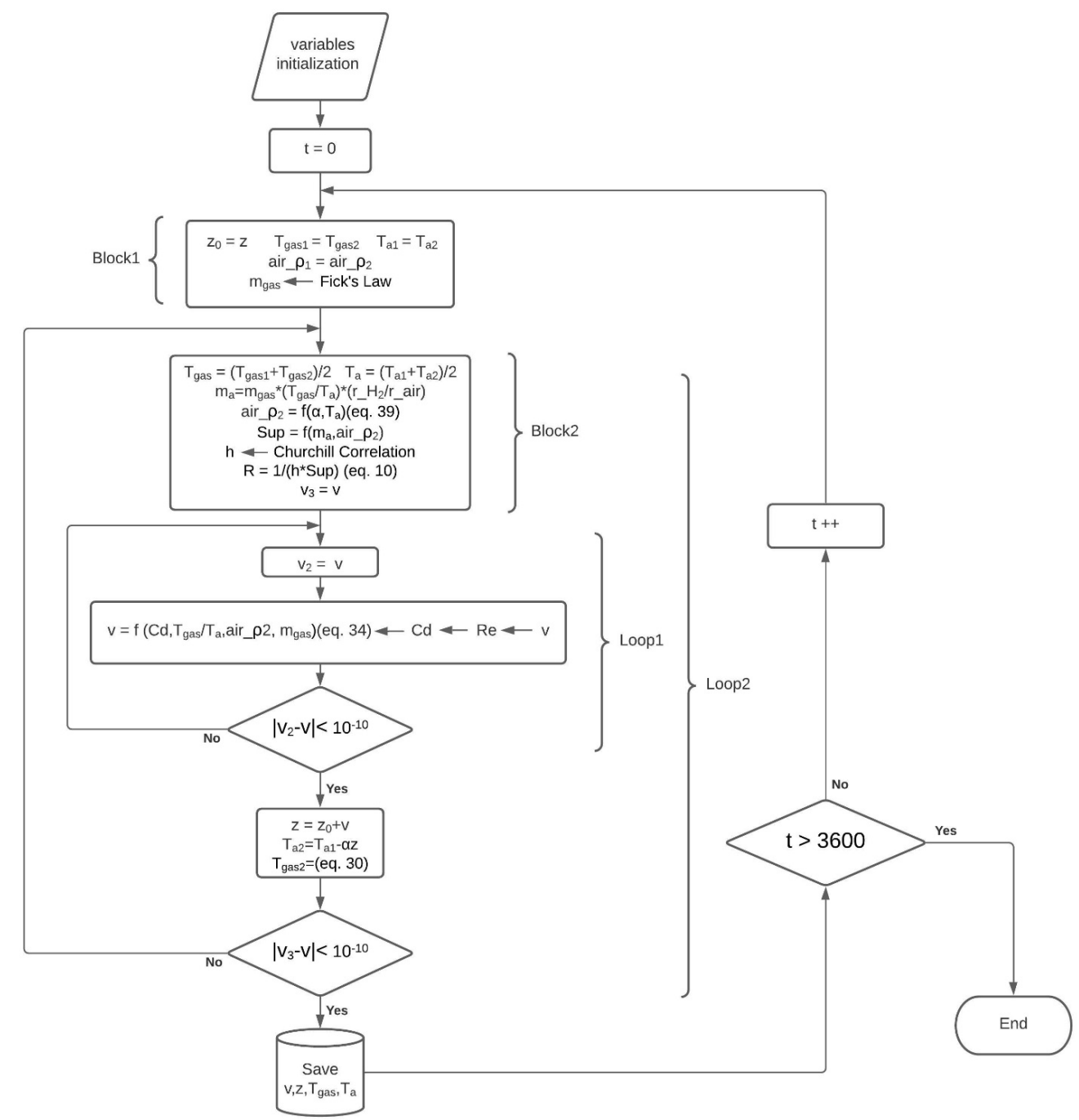

Figure 8: Program flow chart.

The program calculates the ascent rate according to equation 34 at one-second time intervals. However, the ascent rate depends on the drag coefficient which, in turn, depends on the Reynolds number (equation 4), also dependent on ascent rate (equation 3). Furthermore, at a given time, both the density and the temperature of the surrounding atmosphere $\left(T_{a}\right)$ depend on the altitude reached and, therefore, on the ascent rate. We can state the same about the temperature of the lifting gas $\left(T_{\text {gas }}\right)$ which, in addition to depending on the ascent rate, also depends on the thermal resistance (equation 30). And this resistance depends on the balloon's surface area (equation 10), which is dependent, in turn, on altitude. To sum up, there is a strong connection and interdependence between many of the variables in equation 34, meaning it cannot be solved directly. To solve it we used several iterative steps following the outline represented in Fig. 8 . 
The aim of the program is to use equations found and described in earlier sections to calculate the balloon's ascent rate and altitude and the temperature of the lifting gas at one-second intervals, starting out from initial ground conditions of temperature, density and vertical temperature gradient $(\alpha)$ at launch time. Once the ascent rate has been calculated for each time interval (equation 34), we can determine the gain in altitude and, with the value of $\alpha$ for that interval, we can find the variations in air temperature and density using equation 39. We shall now explain the tasks carried out for each of the blocks shown in Fig. 8.

After introducing constants and initializing variables (density, temperature, etc.), a counter is activated which increases variable $t$ by one second at a time until it reaches $3600 \mathrm{~s}$. This counter includes all the blocks where the principal calculations are developed. Variables of temperature, altitude and density are updated in block 1 based on calculations made at the previous interval. After this, the mass of the lifting gas which escapes by diffusion is calculated using Fick's law and the loss rate of lift found by Terluic et al. (1983). according to the level reached. This allows us to determine the balloon's volume, diameter, and surface area. The Churchill correlation is used to find the heat transfer coefficient $(h)$, which then allows us to find the thermal resistance between the balloon skin and the surrounding air (equation 10).

Loop1 is used to calculate iteratively the ascent rate, the Reynolds number and the drag coefficient at each level. At $t=0$, when the balloon is still on the ground, this loop will calculate the ascent rate at which the drag force and free lift will be in equilibrium for the initial conditions of air density and the balloon's diameter, without taking into account the delay produced by the inertia effect caused by the mass of the gas and balloon skin. The initial conditions mean this initial velocity is 0 , like the $R e$, and this means that the drag coefficient, $C d$, is at its maximum value. Thus, the ascent rate calculated after the first iteration will be low. In the second iteration, as $R e$ increases and $C d$ falls, the new velocity will have a higher value, meaning that after the next iteration $C d$ will be even lower. This process will continue until the convergence of the variables produces an error in the calculation of the ascent rate lower than $10^{-10} \mathrm{~m} / \mathrm{s}$. The resulting value for the ascent rate will coincide with the increase in altitude $\Delta z$ as the time intervals are of 1 second. Knowing the new altitude, we can determine the new temperatures of the surrounding air and lifting gas (equation 30).

However, loop1 calculates ascent rate without updating the altitude and the adiabatic cooling of the lifting gas, which will decrease the volume of air displaced, the free lift, and the surface area of the balloon - factors which also have an influence in the calculation of the heat transfer coefficient $(h)$ and the thermal resistance $(R)$. For this reason, we created a new loop 630 (loop2) to recalculate iteratively the balloon's ascent rate taking into account the heat exchange with the surrounding air. 631 Loop2 will end when, considering all the processes at play, the convergence of the variables results in an error smaller than $10^{-10} \mathrm{~m} / \mathrm{s}$ in the calculation of the velocity.

\section{Ascent rate and thermal jump calculated as a function of the values of different parameters}

635 Applying the model, we investigated how the ascent rate of a $30 \mathrm{~g}$ balloon with a free lift of $140 \mathrm{~g}$ behaves with different 636 lapse rates and what the effect would be of using helium instead of hydrogen. We also studied the magnitude of the effect of the thermal jump in the evolution of the ascent rate. 
The mass of displaced air is virtually constant, although it can decrease slightly due to the adiabatic cooling of the lifting gas. If we assume there are no effects of heat released from the balloon skin itself, and that, for example, the lapse rate of the first few hundred metres of ascent is close to the adiabatic lapse rate for dry air $(g / C p)$, then the temperature of the lifting gas will evolve at the same rate as that of its environment and the mass of displaced air will be constant, as will the lift. If, on the other hand, the lapse rate is lower than, for example, that of a standard atmosphere $\left(6.5 \mathrm{~K} \mathrm{~km}^{-1}\right)$, then a cooling of the gas with respect to the surrounding air will take place which will reduce the balloon's volume and mass of displaced air and, therefore, the lift. However, in this case, as seen in the graph in Fig. 9, even though the balloon has less lift, it will rise faster than in the case of a dry adiabatic lapse rate. Why? The explanation is because the temperature drops with height at a slower rate, at a certain altitude the air will be warmer and this will produce a lower density which will decrease the drag force - and this has more effect than a relatively low free lift.
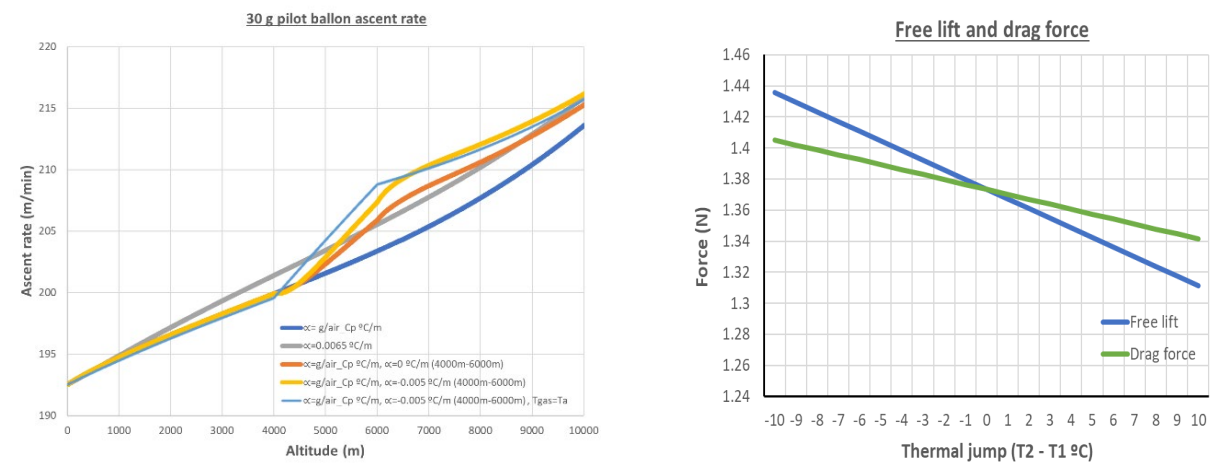

Figure 9: (left) Ascent rate for a $30 \mathrm{~g}$ balloon if $\alpha$ is that of a standard atmosphere (grey line) or if $\alpha$ coincides with the adiabatic lapse rate of dry air (dark blue line). Similar case but with an isothermal layer between $4 \mathrm{~km}$ and $6 \mathrm{~km}$ altitude (red line), or with an inversion layer at the same altitude (orange line) and the case (light blue line) without taking into account the effect of the thermal jump.

Figure 10: (right) Free lift and drag force as a function of the thermal jump, assuming a constant ascent rate equal to that when the two forces are in equilibrium with no thermal jump.

The drag force is proportional to the transversal surface area (equation 2) and, therefore, to the square of the balloon's radius - which increases with altitude - but also to the air density, and, therefore, to the inverse of the cube of the radius (if we consider displaced mass to be virtually constant). The increase in ascent rate seen as the balloon gains height in all the cases in Fig. 9 is down to the consequent drop of the air density. If we consider a hydrostatic equilibrium, the more slowly temperature drops with height, the more quickly the density will fall, meaning the drag force will also fall more quickly, and the ascent rate will increase faster. the case of an isothermal layer (red curve) or an inversion layer (orange curve) between $4 \mathrm{~km}$ and $6 \mathrm{~km}$ altitude. An acceleration of the ascent rate is seen in both cases but it is greater for the inversion layer as in this case density falls more quickly with height. However, why does the ascent rate not drop to the level corresponding to its lapse rate $(g / C p$ in this 
case) after $6 \mathrm{~km}$ altitude? Actually, it does - but slowly. This is due to the fact that once the balloon has reached a greater velocity, the regime becomes more turbulent, the $R e$ increases, the drag coefficient falls and a balance between drag force and free lift at a higher ascent rate is established.

To see the effect the cooling of the lifting gas has on the evolution of the ascent rate, we calculated the ascent rate for the case of an inversion layer between $4 \mathrm{~km}$ and $6 \mathrm{~km}$ altitude, forcing an equality between surrounding air temperature and lifting gas temperature ( $T_{g a s}=T_{a}$ ) (thin blue curve). The delay between the orange curve and this blue one is due to the time needed for the cooling of the lifting gas: for a specific altitude (between $4 \mathrm{~km}$ and $6 \mathrm{~km}$ ), the orange curve presents lower ascent rate values due to the loss of lift thanks to the relative contraction of the balloon. This is caused by a lower lifting gas temperature and the consequent decrease in mass of displaced air. For a specific altitude and air density, the reduction in the balloon's radius caused by the thermal jump (difference between surrounding air temperature $T_{a}$ and lifting gas temperature $T_{\text {gas }}$ ) will lower the drag force, but less than the free lift. Therefore, the ascent rate at which both forces will be in equilibrium will be lower, the higher the thermal jump and vice versa (Fig. 10).

Thermal jumps calculated for the above-mentioned cases are shown in Fig. 11. The dark blue curve shows that the thermal jump for a gradient equal to the adiabatic lapse rate of dry air is $-1{ }^{\circ} \mathrm{C}$. That is, the gas temperature is higher than the surrounding air temperature. This is due to the fact that the model takes into account the thermal capacity of the balloon envelope and that it is the residual heat contained in this skin that, when it dissipates as the balloon rises, maintains the balloon $1{ }^{\circ} \mathrm{C}$ warmer than its surrounding environment. The red and orange curves show the thermal jump for cases with an isothermal layer or an inversion layer between $4 \mathrm{~km}$ and $6 \mathrm{~km}$ altitude. The inversion layer situation gives the largest thermal jump (almost $4{ }^{\circ} \mathrm{C}$ ), as expected from equation 25 . This happens as when there is inversion, more thermal power must enter from outside through the thermal resistance (Fig. 7) - on the one hand, to provide the power needed for the expansion of the gas, and, on the other hand, to inject internal energy into the gas because as it rises, so must its internal temperature in accordance with the outer temperature.

694 in Fig. 11) for the case of an inversion layer. As expected, there is no thermal jump except for that seen at the altitudes of the inversion layer. Furthermore, the thermal jump is lower than it would be in a case where we do not rule out the

698 balloon's mass as maintaining a free lift of $140 \mathrm{~g}$ without adding the $30 \mathrm{~g}$ of the balloon means the mass of lifting gas is 
701

702

703

704

705

706

707

708

709
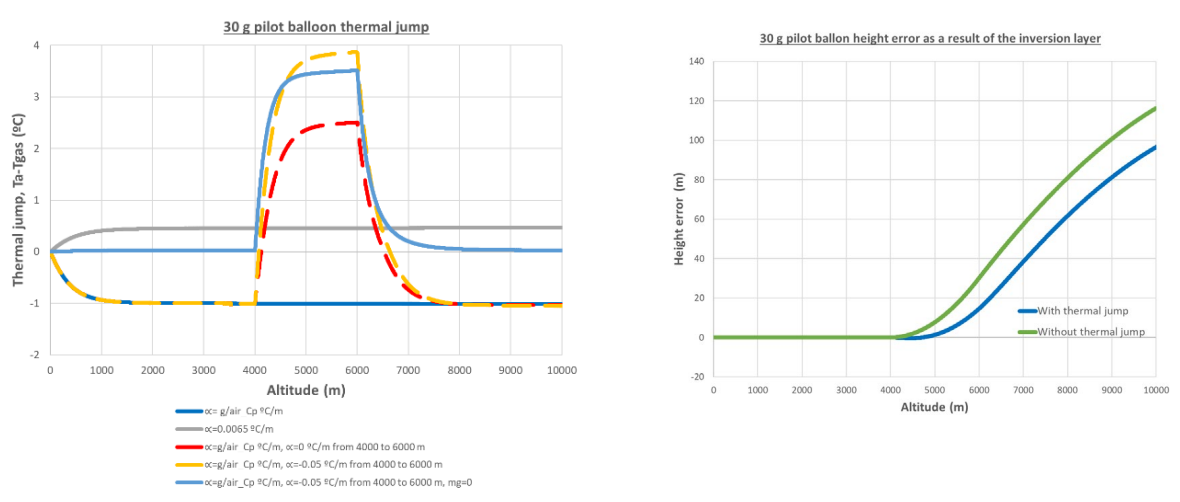

Figure 11: (left) Thermal jump (Ta-Tgas) for a $30 \mathrm{~g}$ balloon for $\alpha$ in a standard atmosphere (in grey), or $\alpha$ coinciding with adiabatic lapse rate of dry air (dark blue line). Similar case but with an isothermal layer between $4 \mathrm{~km}$ and $6 \mathrm{~km}$ altitude (red line), or an inversion layer (orange line), ignoring the mass of the balloon skin (light blue line), and for helium gas (fine green line).

Figure 12: (right) Difference between height reached for $30 \mathrm{~g}$ balloon if lapse rate $\alpha$ coincides with dry air adiabatic lapse rate but with an inversion layer between $4 \mathrm{~km}$ and $6 \mathrm{~km}$ altitude with respect to a case without inversion layer. Taking thermal jump into account (blue line), or not (green line).

Fig. 12 shows the difference between the altitude reached in the case with an inversion layer compared to the altitude reached if the lapse rate were constant - the blue curve represents a situation considering the thermal jump whereas the green one represents the case where we ignore it. As seen previously in Fig. 9, if we do not take into account the heat exchange between lifting gas and surrounding environment (without a thermal jump), acceleration will be instantaneous when an inversion layer is reached, with an abrupt effect on the ascent rate. However, in reality the delay in this response due to a cooling of the lifting gas compensates to some extent the effect the inversion layer has on the ascent rate, thus reducing the error in estimating the altitude of the pilot balloon with respect to a case assuming a constant temperature lapse rate.

For the case with a standard atmosphere $\left(\alpha=6.5 \mathrm{~K} \mathrm{~km}^{-1}\right)$, we compared the height reached with no thermal jump and that reached with the actual thermal jump computed from the model. The difference is very small, with the former being about $8 \mathrm{~m}$ higher than the latter at an altitude of $10 \mathrm{~km}$. This extremely small difference indicates that, at least, for this kind of ( $30 \mathrm{~g})$ balloon, the heat exchange with the surrounding air has very little effect on the ascent rate and is, therefore, virtually insignificant for calculating wind velocities at height.

We carried out an experiment for a case of an inversion layer between $4 \mathrm{~km}$ and $6 \mathrm{~km}$ altitude changing the hydrogen gas for helium while maintaining a constant free lift of $140 \mathrm{~g}$. As the molecular weight of helium is almost twice that of hydrogen (helium is monatomic whereas hydrogen is diatomic), with equal pressure, volume and temperature, there is almost double the mass of helium (same number of atoms and molecules), meaning the free lift will be lower. This means that to maintain the free lift, we must add more mass (and volume) of helium, increasing the surface and drag force. For this reason, ascent rates for helium balloons are lower (Fig. 13), and consistent with reference values used by the US 

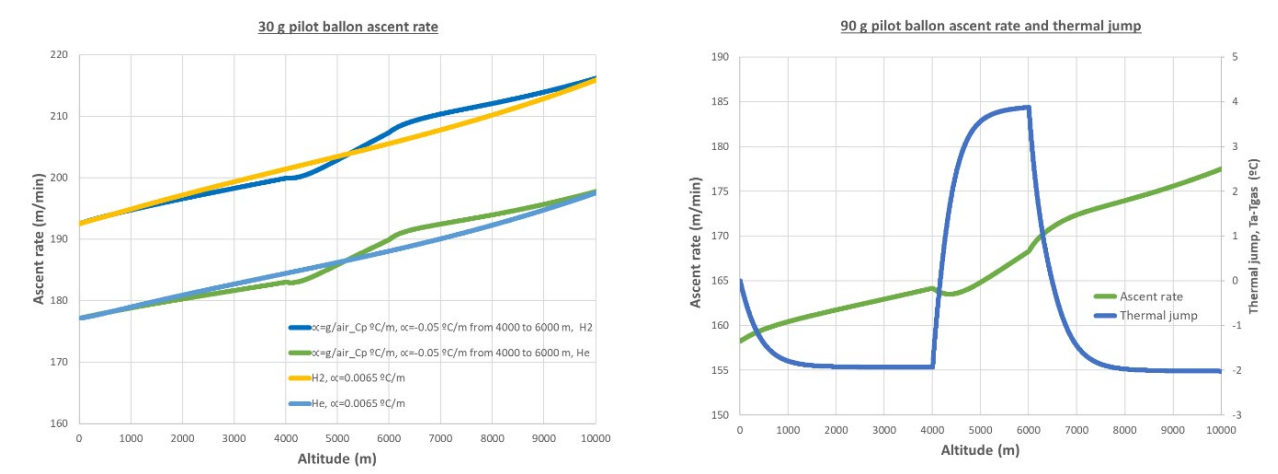

Figure 13: (left) Ascent rate for $30 \mathrm{~g}$ balloon and $140 \mathrm{~g}$ free lift, if $\alpha$ coincides with dry air adiabatic lapse rate in the case of an inversion layer between $4 \mathrm{~km}$ and $6 \mathrm{~km}$ altitude and lifting gas is hydrogen (dark blue line) compared with ascent rate for helium (green line). Also, for a case of $\alpha=6.5 \mathrm{~K} \mathrm{~km}^{-1}$ if gas is hydrogen (orange line) or helium (light blue line).

Figure 14: (right) Thermal jump (blue line) and ascent rate (green line) for a $90 \mathrm{~g}$ balloon inflated to a free lift of $110 \mathrm{~g}$, if $\alpha$ coincides with dry air adiabatic lapse rate and inversion layer between $4 \mathrm{~km}$ and $6 \mathrm{~km}$ altitude.

To analyse the dynamic behaviour of larger balloons, we calculated the ascent rate and thermal jump for a $90 \mathrm{~g}$ hydrogen balloon with a free lift of $110 \mathrm{~g}$ for a dry air adiabatic lapse rate and an inversion layer between $4 \mathrm{~km}$ and $6 \mathrm{~km}$ altitude (Fig. 14). We chose this balloon and lift because the Reynolds number here is far from the critical zone and is within the domain where the formula we found empirically above (equation 4) is still valid for calculating the drag coefficient, and furthermore at the Ebro Observatory and other observatories around the country, such a combination was common over several decades. In this case, the thermal jump outside of the inversion layer is quite negative (almost $-2{ }^{\circ} \mathrm{C}$ ). Owing to the increased thermal capacity of the balloon skin, more heat per unit of time is released as the balloon rises, keeping the lifting gas relatively warm and contributing to an increase in its lift. However, this changes abruptly at $4 \mathrm{~km}$ altitude where the thermal jump rises by over $6{ }^{\circ} \mathrm{C}$, causing a sudden loss of lift which leads to a sudden drop in the ascent rate, However, the large fall in air density as the balloon rises within the inversion layer will, as we explained earlier, progressively increase the ascent rate due to the decrease in drag force. Once we reach an altitude of $6 \mathrm{~km}$, this process is reversed. But the most relevant aspect in this case, is the low ascent rate with regards to the former $30 \mathrm{~g}$ hydrogen balloon inflated to $140 \mathrm{~g}$ free lift. In this case, the average ascent rate along its vertical route is far lower than $200 \mathrm{~m} \mathrm{~min}^{-1}$ (an average of $167 \mathrm{~m} \mathrm{~min}^{-1}$ from ground level to $10 \mathrm{~km}$ altitude), and this was an important source of error if the same constant rate of $200 \mathrm{~m} \mathrm{~min}^{-1}$ was assumed, as is indeed the case. Furthermore, such a lower ascent rate was to be expected, taking into account that two parameters point in the same direction: a lower free lift and a greater weight of the rubber skin. the correlations obtained between wind speeds calculated with the model or from ERA5 barely vary. In fact, the altitude profile obtained with this $C d$ value is very close to that obtained calculating $C d$ in the way we explained previously. This can be seen in Fig. 15, although the average value of $C d$ for the vertical evolution of the balloon is a little lower (Fig. 16). 

similar to the case with an ascent rate of $220 \mathrm{~m} \mathrm{~min}^{-1}$.
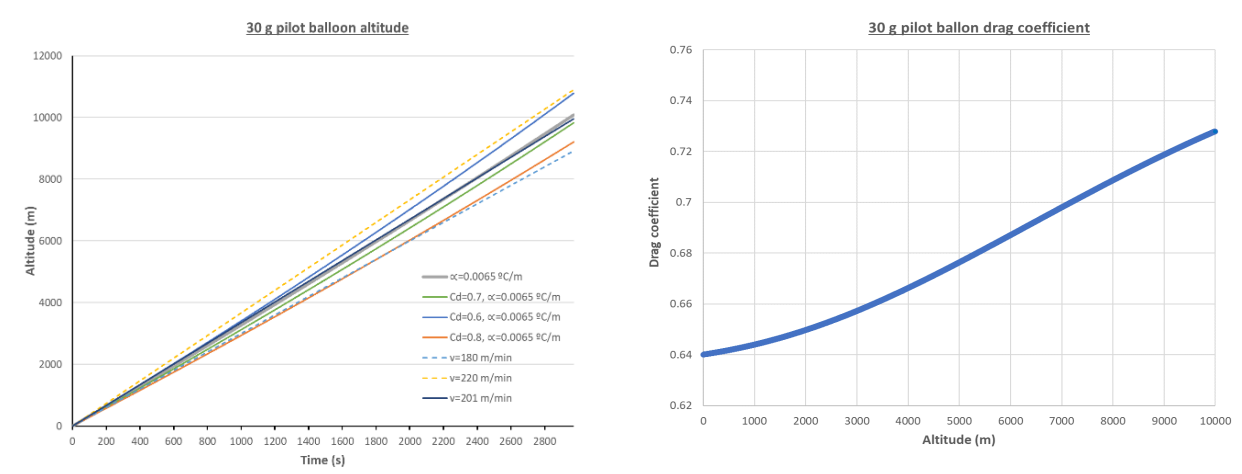

\section{Application of the model to the case of a balloon at rest}

Figure 15: (left) Altitude profile versus time for a standard atmosphere temperature lapse rate computed from the model (grey), forcing a constant $C d$ of 0.7 (green line), 0.6 (blue line), 0.8 (orange line). Altitude profile for different constant vertical ascent rates: $180 \mathrm{~m} \mathrm{~min}^{-1}$ (dotted blue line), $220 \mathrm{~m} \mathrm{~min}^{-1}$ (dotted orange line) and $201 \mathrm{~m} \mathrm{~min}^{-1}$ (dark blue line).

Figure 16: (right) Drag coefficient as a function of altitude computed from the model.

The constant ascent rate which generates the altitude profile most similar to that produced by the model is $201 \mathrm{~m} \mathrm{~min}^{-1}$ (Fig. 15). This confirms that the estimate of $200 \mathrm{~m} \mathrm{~min}^{-1}$ used in the past for the $\mathrm{EO}$ observations using $30 \mathrm{~g}$ balloons filled with hydrogen with $140 \mathrm{~g}$ free lift was an accurate one.

To check our hypothesis that the sphere is isothermal and the temperature gradient is principally found between the balloon's rubber envelope and the surrounding air, we carried out a simulation forcing the initial temperature of the lifting gas to be $40^{\circ} \mathrm{C}$, the air, $22^{\circ} \mathrm{C}$, and an ascent rate of 0 . This allowed us to calculate the rate at which the balloon will cool if it stays at rest. The values we obtained (red curve, Fig. 5) are consistent with the thermal jumps found previously (blue curves, Fig. 5). This supports the idea that the greatest part of the thermal jump is to be found between the balloon skin and the surrounding air. In a case where the lifting gas represents a significant degree of resistance for heat transfer, we would have observed a slower cooling than in the calculated case assuming an isothermal sphere and applying the Churchill correlation in a model where the only thermal resistance is found between the skin and the surrounding air. Given the high level of consistency between the observed and calculated cooling rate, we can state that our initial hypothesis is valid. response of the thermal system. In this case, as we expected, the balloon cooled much more quickly as can be seen in the 

to climate issues but also to enable us to test whether our thermodynamic model can improve the precision of wind speed measurements at high altitudes with regards to the assumption of a constant ascent rate. To this end, we selected a 10-day sample for observations when the balloon reached or rose above $10 \mathrm{~km}$ altitude. We chose this altitude as it is high enough to offer a better detection of any errors in the calculation of the wind speed. The altitude is calculated by integrating the ascent rate over time. Thus, regarding possible errors in the ascent rate, the higher the altitude, the greater the accumulated error when estimating the altitude and this will be seen through a greater error in the calculation of wind speed.

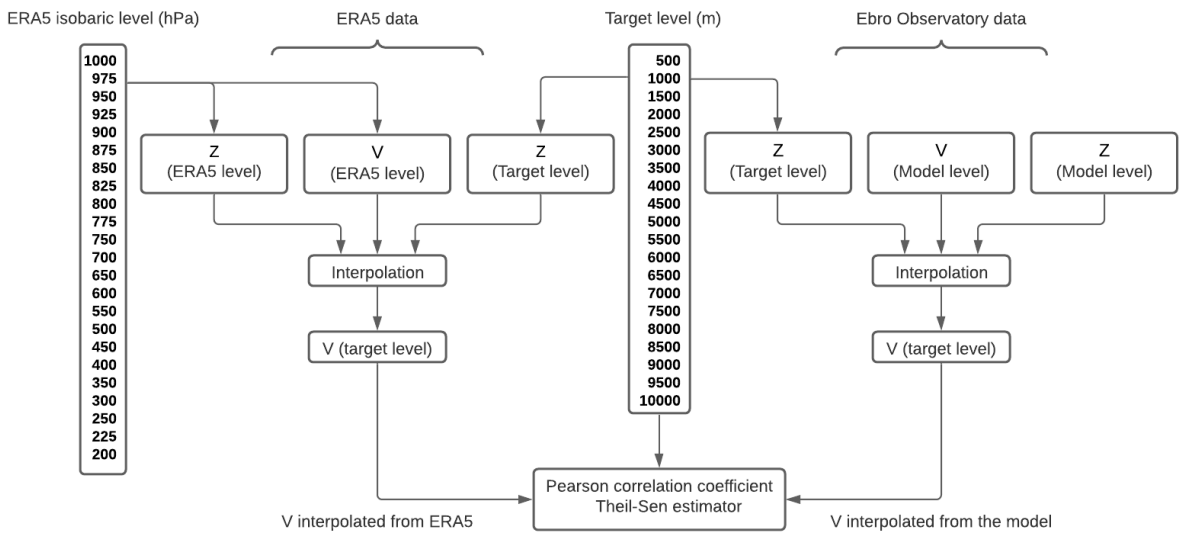

804

Figure 17: Interpolation process scheme.

For the group of days in our sample, we used wind data from the ERA5 reanalysis to be able to compare them with wind speeds obtained from the thermodynamic model applied to data obtained at the Observatory. However, these data refer to pre-established isobaric levels whereas wind data from EO observations refer to altitudes estimated by the thermodynamic model, corresponding to specific time intervals when the observers took systematic measurements of the azimuth and elevation angles. These time intervals were of 30 seconds each up until 5 minutes and then, after that moment, one-minute intervals were used. Since the wind data from the two sources (observations and ERA5) refer to different altitudes, we carried out an interpolation process (Fig. 17), calculating these data for a range of altitudes between $500 \mathrm{~m}$ $-10 \mathrm{~km}$ at 500-metre intervals. For each day and each data group (ERA5 and observations) we obtained a unidimensional matrix with 20 values for each of the two wind components, corresponding to the 20 pre-established altitudes $(500 \mathrm{~m}$ $10 \mathrm{~km}$ ). Finally, with the matrixes of each of the 10 days in the sample, we set up, for each wind component, a unidimensional matrix of 200 elements $(10 \times 20)$ for the data from observations applying the thermodynamic model and another one for the ERA5 data. We made several statistical calculations using these values. 
Table1: Correlation coefficients between values calculated using thermodynamic model and values calculated using ERA5

We calculated the Pearson correlation coefficient and the Theil-Sen estimator for both wind components (zonal and meridional) (table 1). We found the best results using data corresponding to the meridional component (Fig. 18), and in both cases we found that the values obtained with the thermodynamic model are greater than those corresponding to the reanalysis. The thermodynamic model data are calculated from measurements taken at a specific geographical point, giving a more direct vision of the size of the vertical profile of the wind at this specific point. The values obtained from the reanalysis, though, are based on a larger scale and calculated using the interpolation of observations taken at geographical points far from the Ebro Observatory. Taking into account this interpolation process, although the reanalysis model has a very good spatial resolution, it was to be expected that values taken from reanalysis would be slightly lower.
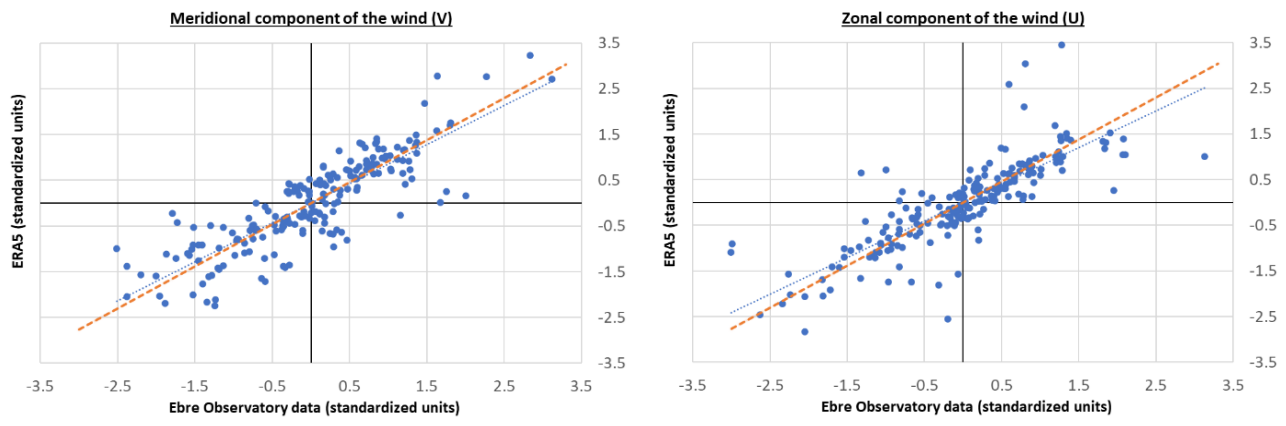

Figure 18. Meridional (left) and zonal (right) wind components calculated with ERA5 compared to case calculated with thermodynamic model and EO observations. Regression line for minimum squares (blue line) and Theil-Sen estimator (orange line).

To analyse the degree of improvement in the final result thanks to using the thermodynamic model, we used other hypotheses evaluating their influence on estimates of the balloon's altitude as a function of time. In this way, to contrast the values obtained using the thermodynamic model, we calculated the profile of the balloon's altitude as a function of time for different constant ascent rates. From the new altitude profiles, we obtained and the observation data from the azimuth and elevation angles, we recalculated and interpolated the wind speeds at height at the EO for each case. The results are shown in Fig. 19. This graph shows, for each of the wind components, the correlations obtained between the group of wind data from ERA5 and the wind data (200 values in each group) from calculations based on observations using different hypotheses for the ascent rate. As expected, the correlations fall when the ascent rates are too slow (less than $190 \mathrm{~m} \mathrm{~min}^{-1}$ ) or too fast (over $210 \mathrm{~m} \mathrm{~min}^{-1}$ ). Although the values obtained around $200 \mathrm{~m} \mathrm{~min}^{-1}$ are not very different, 


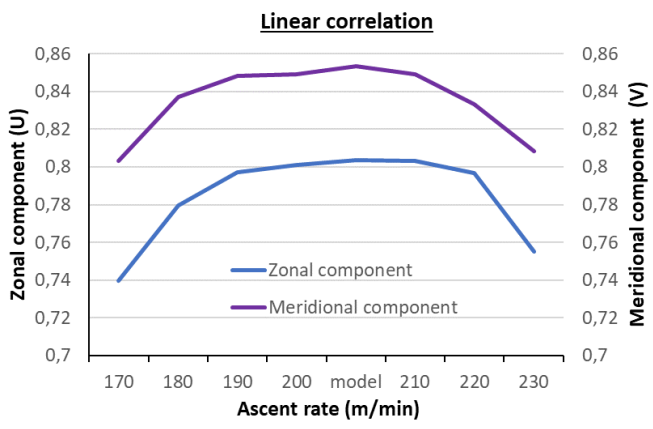

Figure 19: Linear correlation as a function of the altitude profile corresponding to each ascent rate hypothesis.

Having noted this, the inherent errors in the azimuth and elevation angle measurements (for example, delays or advances in taking measurements in each time interval) means that the data from pilot balloon observations do not allow us to make a more precise assessment of the thermodynamic model. For example, correlations obtained between the ERA5 wind profile and the model hardly vary even when using the lapse rate for a standard atmosphere $\left(6.5 \mathrm{~K} \mathrm{~km}^{-1}\right)$ for all days of the sample, with respect to the case of using the corresponding lapse rate obtained from ERA5 for each of the sample days. On the other hand, in practice, it is difficult to launch balloons with a precisely determined rate of ascent. Thus, where there is significant vertical shear at low levels, possibly associated with significant differences in vertical velocity from thermals, pilot-balloon measurements could be adversely affected by height assignment errors. (Guide to Meteorological Instruments and Methods of Observation, 2014). Furthermore, even with the relatively good resolution the reanalysis model offers, it is possible that this may not provide the required precision on a local level. All these factors contribute to explaining the small difference in the correlations we obtain using different hypotheses (Fig. 19).

\section{Conclusions}

One of the most significant causes of errors in the measurement of altitude winds using a pilot balloon and an optical theodolite occurs when recording altitudes for the balloon as a function of time (WMO-No.8, 2008). The balloon's altitude at each time interval when observers measure the azimuth and elevation angles depends on the evolution of the ascent rate which changes over time. For this reason, it is essential to determine this variable as accurately as possible. has an average ascent rate of around $200 \mathrm{~m} \mathrm{~min}^{-1}$ during the first $10 \mathrm{~km}$ of ascent. The ascent rate is not constant, however, but sees an increase of $10 \%$ over its initial value. This positive acceleration is down to different reasons. The principle reason is the decrease in atmospheric density with altitude which means a decrease in drag force although, as altitude is gained, the drag coefficient $C d$ becomes larger. The drag coefficient increases with height because the Reynolds number, $R e$, decreases, causing the aerodynamic regime to become less and less turbulent. The Reynolds number decreases with altitude although it is proportional to the balloon diameter, which increases with altitude thanks to the progressive drop

884 in atmospheric pressure. The reason why Re decreases is because it is also proportional to air density and this decreases more quickly with altitude than the speed at which the diameter expands, and so the overall effect reduces $R e$. 

rate. We calculated that there is around a $20 \mathrm{~m}$ loss of height caused by diffusion at altitudes of $10 \mathrm{~km}$.

We found that the difference between the temperature of the air surrounding the balloon and the lifting gas temperature (the thermal jump) will be greater, the greater the ascent rate, the larger the thermal resistance between the balloon skin and environment, and the greater the mass of the lifting gas, but also the more slowly temperature drops with altitude. Thus, with an isothermal layer or an inversion layer, a larger thermal jump is expected. On the other hand, for high temperature lapse rates, as would be the case for the dry atmosphere adiabatic lapse rate, the thermal jump would be negligible. In our specific case, for the $30 \mathrm{~g}$ balloons used by the Ebro Observatory, we found that the thermal jump is very low even when assuming natural convection. Thus, we can expect that this thermal jump will be even lower as the convection is actually a forced one, and that it will have a negligible effect on the ascent rate - or at least regarding the calculation of the vertical wind speed profile using an optical theodolite.

We have also seen that helium-filled balloons rise at a lower ascent rate than hydrogen ones because the atomic mass of helium is greater than the molecular mass of hydrogen. This means that helium balloons must displace more air to obtain a specific free lift, and this implies a larger diameter and, therefore, an increase in drag force and a decrease in the ascent rate. Ascent rate values calculated for $30 \mathrm{~g}$ helium-filled balloons with a $139 \mathrm{~g}$ free lift are consistent with the values used by the US National Weather Service. We also saw that for the same temperature lapse rate conditions, and assuming equal ascent rates, we should expect a greater thermal jump for helium balloons because helium is monatomic, meaning its capacity for storing internal energy is lower than that of hydrogen and its adiabatic lapse rate is greater than that of hydrogen and, therefore, of the air. However, this increase in the thermal jump is compensated for by the fact that helium balloons have a lower ascent rate as we have seen.

Thanks to the digitized data base at the Ebro Observatory, we were able to calculate the meridional and zonal wind components at altitude assuming different ascent rate hypotheses for a sample of days in which the balloons reached a height of $10 \mathrm{~km}$, and we compared these results with those obtained from the ERA5 reanalysis. Results indicate that in the case of a constant ascent rate, the value of $200 \mathrm{~m} \mathrm{~min}^{-1}$ estimated at the Observatory in the past for $30 \mathrm{~g}$ hydrogen balloons inflated to $140 \mathrm{~g}$ free lift was quite accurate. However, this value is too high for the hydrogen balloons weighing approximately $90 \mathrm{~g}$ inflated to around $110 \mathrm{~g}$ free lift used at the Ebro Observatory and other observatories around the country, such the Meteorological Observatory of Badajoz during the 1920s and the early 1930s. We do not know for sure the reasons why this type of balloons was replaced by the $30 \mathrm{~g}$ ones inflated to $140 \mathrm{~g}$ free lift, but it may have been because of the progressive improvement of the materials used in the manufacture of the rubber skin and for a better consistency with the assumed $200 \mathrm{~m} \mathrm{~min}^{-1}$ ascent rate. theodolite, as these observations are also influenced by the physical limits of both the device and the observer. Having said that, the thermodynamic model we present may also be useful for other applications that do require a better knowledge 
This research would not have been possible without the efforts of the Ebro Observatory observers. All of them deserve our highest recognition for their hidden and quiet, but efficient, work. The authors extend their appreciation to the National Center for Atmospheric Research (NCAR) for their powerful and versatile software (NCL) and also to the many people who have invested huge amounts of time and energy making $R$ the great software that we have been able to use. The results of this work contain modified Copernicus Climate Change Service information 2021. Neither the European Commission nor ECMWF is responsible for any use that may be made of the Copernicus information or data it contains.

\section{Bibliography}

935

Alexander, P., A numerical study of open atmospheric balloon dynamics, Physics of Fluids, 15, 3065, 2003. doi: 10.1063/1.1607325.

Atkinson, Kendall E. (1989), An Introduction to Numerical Analysis (2nd ed.), New York: John Wiley \&

939 Bell, B., Hersbach, H., Berrisford, P., Dahlgren, P., Horányi, A., Muñoz Sabater, J., Nicolas, J., Radu, R., Schepers, D.,

940 Simmons, A., Soci, C., Thépaut, J-N. (2020): ERA5 hourly data on pressure levels from 1950 to 1978 (preliminary

941 version). Copernicus Climate Change Service (C3S) Climate Data Store (CDS). (Accessed on < 21-04- 2021>),

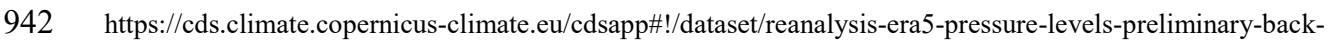

943 extension?tab=overview

944 Boatman, The Effect of Tropospheric Temperature Lapse Rates on the Ascent Rates of Pilot Balloons, J. Appl. Meteor., $94513,955,1974$.

946 Churchill, S.W., Comprehensive theoretically based, correlating equations for free convection from isothermal spheres, 947 Chem. Engng Commun. 24:4-6, 339-352, 1983. doi: 10.1080/00986448308940090

948 Conrad, G.R., Robbins, E.J., Determination of balloon drag. AIAA PAPER 91-3666, 1991.

949 Cross, R., Aerodynamics of a Party Balloon, The Phys. Teacher, Vol. 45, 334, 2007. doi: 10.1119/1.2768686.

950 Crouch, T.D., Lighter than air. Johns Hopkins University Press, 2008, ISBN 978-0-8018-9127-4.

951 Etherington, H., Nuclear Engineering Handbook. McGraw-Hill, Section 9-2, 1958.

952 Fichtl, G.H., DeMandel, R.E., Krivo, J.K., Aerodynamic Properties of Spherical Balloon Wind Sensors, J. Appl. Meteor., $95311,472,1972$.

954 Gallice, A., Wienhold, F.G., Hoyle, C.R., Immler, F., Gallice, T.P., Modeling the ascent of sounding balloons: derivation 955 of the vertical air motion, Atmos. Meas. Tech., 4, 2235, 2011.doi:10.5194/amt-4-2235-2011.

956 Guide to Meteorological Instruments and Methods of Observation: (CIMO guide). 2014 edition. 957 (http://dx.doi.org/10.25607/OBP-432)

958 Jasperson, W.H., The limiting accuracy of wind profiles obtained by tracking rising balloons, J. Appl. Meteor, 21 (6): 959 816, 1982. doi:10.1175/1520-0450.

960 Komsta, L. (2013), Median-Based Linear Models. R package version 0.12.1 http://CRAN.R-project.org/package=mblm

961 Knowles, W.E., Invention of the Meteorological Instruments, The Johns Hopkins Press, Baltimore, 1969.

962 Luers, J.K., MacArthur, C.D., The limitations of winds measurements accuracy for balloons systems, Journal of Applied 963 Meteorology, 13, 1, 168, 1974.

964 Mapleson, W.W., The drag of spherical rubber balloons, Quart. J. Roy. Meteorol. Soc. 80, 449, 1954. 965 doi.org/10.1002/qj.49708034517. 
966 Meteorological Office, Handbook of Meteorological Instruments, Part II: Instruments for Upper Air Observations.

967 Published by HMSO. London. 1961.

968 Nelson, R.A., Comments on "The Effect of Tropospheric Temperature Lapse Rates on the Ascent Rates of Pilot Balloons", 969 J. Appl. Meteor., 14, 1608, 1975.

970 Perkins, D.T., The Response of Balloons to the Wind, Bull. Amer. Meteor. Soc. 33 (4): 135, 1952. Doi: 10.1175/1520971 0477-33.4.135.

972 Puig, I., El Observatorio del Ebro: idea general sobre el mismo. Imp. Moderna del Ebro de Algueró y Baiges, Tortosa, 9731927.

974 R Core Team (2020). R: A language and environment for statistical computing. R Foundation for Statistical Computing,

975 Vienna, Austria. URL https://www.R-project.org/.

976 Saint-Exupéry, A., Courrier Sud, NRF, Gallimard, France, 1929.

977 Scoggins, J.R., Aerodynamics of Spherical Balloon Wind Sensors, J. Geophys. Res., 694, 591, 1964.

978 Scoggins, J.R., Spherical Balloon Wind Sensor Behavior, J. Geophys. Res., 4, 139, 1965.

979 Scoggins, J.R., Sphere behavior and the measurement of wind profiles. NASA Tech. Note D-3994, Marshall Space Flight 980 Center, Huntsville, Ala., 53 pp., 1967.

981 Sen, P.K. (1968). Estimates of Regression Coefficient Based on Kendall's tau. J. Am. Stat. Ass. 63, 324, 1379-1389.

982 Terliuc, B., Asculai, E., Doron, E., The Use of Compensated Aerological No-Lift Balloons to Determine Relatively Long-

983 Term Dry-Air Parcel Trajectories, J. Climate Appl. Meteor. 22 (10), 1811, 1983. doi: 10.1175/1520-0450

984 The NCAR Command Language (Version 6.6.2) [Software]. (2019). Boulder, Colorado: UCAR/NCAR/CISL/TDD. 985 http://dx.doi.org/10.5065/D6WD3XH5

986 Theil, H. (1950), A rank invariant method for linear and polynomial regression analysis. Nederl. Akad. Wetensch. Proc. 987 Ser. A 53, 386-392 (Part I), 521-525 (Part II), 1397-1412 (Part III).

988 Wilkinson C., Stefan Brönnimann, Sylvie Jourdain, Emeline Roucaute, Rick Crouthamel \& IEDRO Team, Philip Brohan,

989 Antonia Valente, Yuri Brugnara, Manola Brunet, Gilbert P. Compo.2019. Best Practice Guidelines for Climate Data

990 Rescue. Copernicus Climate Change Service.Reading, delivered 29 March 2019, 35 pp

991 WMO-No.8, 2008. Guide to Meteorological Instruments and Methods of Observation.

992 WMO, N No $^{\circ} 91,1971$. Compendium of lecture notes for training class III. 